\title{
COSMIC EVOLUTION \\ AND THERMODYNAMIC IRREVERSIBILITY
}

\author{
DAvid LAYZer
}

Department of Astronomy, Harvard University, Cambridge, Massachusetts

\begin{abstract}
This paper seeks to explain and relate three macroscopic arrows of time: the thermodynamic arrow, defined by entropy-generating processes in closed systems, the historical arrow, defined by information-generating processes in certain open systems, and the cosmological arrow, defined by the cosmic expansion.
\end{abstract}

\section{INTRODUCTION}

Several speakers at this conference have referred to the zeroth law of thermodynamics. Cosmology also has its zeroth law. It states that cosmoingists are fermions: no two of them can be in the same state of mind at the same time. Earlier in this conference Dr Narlikar explained how the expansion of the universe introduces an asymmetry into local descriptions of radiation processes. He argued that the use of retarded rather than advanced solutions of Maxwell's equations to describe radiation processes supports steady-state cosmology but contradicts conventional relativistic cosmology. On the other hand, Dr Narlikar's theory does not relate the cosmic expansion directly to thermodynamic irreversibility. It ties the electromagnetic arrow of time firmly to the cosmological arrow, but leaves the thermodynamic arrow suspended in mid-air.

The views I wish to elaborate are rather different. First, I believe that the electromagnetic arrow has nothing directly to do with cosmology, but is determined by the thermodynamic arrow in the manner elucidated by Einstein in $1909^{1}$. Einstein pointed out that the retarded and advanced descriptions of radiation processes occurring in any finite region of spacetime are completely equivalent, but the auxiliary conditions in the two descriptions differ in kind. In the retarded description all macroscopic radiation sources must be specified, while in the advanced description the microscopic absorption processes must be specified in detail. In practice one uses the retarded description because one does not have microscopic information about the absorbing matter. For the same reason, if one wishes to describe an irreversible process such as diffusion or heat conduction at the macroscopic level one must describe it as occurring in the 'forward' 
direction of time. In short, Einstein's argument demonstrates that the asymmetry of macroscopic radiation processes results from precisely those properties of matter in bulk that give rise to other macroscopically irreversible phenomena.

On the other hand, I believe that current discussions of thermodynamic irreversibility, though essentially correct, are incomplete, and need to be supplemented by cosmological considerations. To explain this view, let me recall-very schematically-the sequence of steps leading from a reversible microscopic description of an $N$-body system to an irreversible macroscopic description.

\section{THERMODYNAMIC IRREVERSIBILITY}

The first step is to introduce statistics. Instead of saying that an $N$-body system is in a definite state $k$, specified by $6 N$ coordinates and momenta (or by a state vector $\psi_{k}$ ), we specify a probability distribution $\left\{p_{k}\right\}$ or a density matrix $\rho$. The information associated with this description is defined by

$$
I=S_{\max .}-S
$$

where the entropy $S$ is defined by

$$
S=-\sum p_{k} \ln p_{k} \quad \text { or } S=-\operatorname{Tr}\{\rho \ln \rho\}
$$

In equation $1 S_{\max }$ is the maximum value of $S$ consistent with the macroscopic constraints on the system. It is well known that, by virtue of Liouville's theorem, $S$ is a constant of the motion for an isolated $N$-body system; dynamical evolution neither creates nor destroys information. Therefore the passage to a statistical description does not disturb the temporal symmetry of the description.

The next step is coarse-graining. One combines the microstates $k$ into aggregates $\alpha$ within which macroscopic variables such as the energy do not vary_appreciably. Let $\left\{\bar{p}_{\alpha}\right\}$ denote the set of coarse-grained probabilities and $\bar{\rho}$ the coarse-grained density matrix. With the coarse-grained probability distribution (or density matrix) we associate the coarse-grained entropy $\bar{S}$ and a corresponding information measure $\bar{I}$, which I shall call the macroinformation. The microinformation $I^{\prime}$ and the corresponding entropy $S^{\prime}$ are defined by

$$
\begin{aligned}
& S=\bar{S}+S^{\prime} \\
& I=\bar{I}+I^{\prime}
\end{aligned}
$$

It is easy to show that the microscopic entropy $S^{\prime}$ is just the average entropy of the aggregates $\alpha^{*}$. Since the total information $I$ is constant, any change in the macroinformation of the system is accompanied by an equal and opposite change of the microinformation. But merely introducing a distinction between macroscopic and microscopic information does not of course disturb the temporal symmetry of the description.

* The entropy $S_{\alpha}$ of an aggregate is defined in terms of the conditional probabilities $p(k / \alpha)=p_{k} / \bar{p}_{\alpha}$ and in the averaging process that defines $S^{\prime}$ the quantities $S_{\alpha}$ are weighted by the probabilities $\bar{p}_{\alpha}$. Thus

$$
S^{\prime}=\sum_{\alpha} \bar{p}_{\alpha}\left\{-\sum p(k \mid \alpha) \ln p(k \mid \alpha)\right\}
$$


Temporal asymmetry is introduced by the third and most crucial step $\dagger$. Fifteen years ago van $\mathrm{Hove}^{2}$ proved that if the Hamiltonian of a closed system satisfies a certain rather general condition and if the off-diagonal elements of the coarse-grained density matrix $\bar{\rho}$ vanish at a particular instant, then the coarse-grained entropy $\bar{S}$ will subsequently increase monotonically until it assumes its greatest possible value, when the system will be in a state of thermodynamic equilibrium. The essential feature of van Hove's theorem is that it relates the irreversible increase of entropy in a closed system to a property of the initial state. Several similar theorems have subsequently been proved $^{3}$. All of them state that if microinformation (suitably defined) is initially absent in a closed system, then the macroinformation will subsequently decrease monotonically. The thermodynamic arrow is thus defined by a unidirectional flow of information from macroscopic into microscopic degrees of freedom.

Modern theories of irreversible processes have yielded valuable insight into the detailed mechanisms responsible for the approach to equilibrium, as well as powerful methods for calculating transport coefficients. The following discussion proceeds from the assumption that these theories are essentially correct. But if they are correct, they cannot be complete.

For example, a theory that deals only with closed systems obviously cannot explain why irreversible processes occurring in different closed systems should define the same arrow. Again, the coarse-graining procedure, and hence the dividing line between macroscopic and microscopic information, is largely arbitrary in the theories under consideration. Finally, these theories offer no justification for the assumption that microinformation is initially absent. Indeed, the very meaning of this assumption is unclear. The absence of microinformation in a theoretical description does not implyaccording to currently held views--that it is unattainable in some objective sense, but only that it is uninteresting or hard to get, or both. This seems to imply that thermodynamic irreversibility is at least in part a psychological phenomenon - a position that most physicists would probably be unwilling to accept, though it has been advocated by some.

\section{BOREL'S ARGUMENT}

The recognition that no finite physical system can be truly isolated from the rest of the universe seems at first sight to offer an attractive solution to these difficulties. The Hamiltonian of every ostensibly closed system contains a finite contribution representing the interaction between the system and its environment. So long as we focus attention on a definite system, omitting from our description all dynamical variables referring to particles and fields outside the system, the interaction Hamiltonian is not fully known and hence has a stochastic character. This interaction can have a profound effect on the microscopic state of a system, even if it has been shielded as carefully as possible from outside influences. Thus E. Borel ${ }^{4}$ calculated that moving $1 \mathrm{~g}$ of matter through $1 \mathrm{~cm}$ at the distance of Sirius would destroy

\footnotetext{
$\dagger$ For sake of simplicity, there is here no discussion of the thermodynamic limiting processa matter of considerable technical interest which, however, is not directly relevant to the present discussion.
} 
microinformation about the state of a macroscopic gas in $10^{-6}$ second. Such calculations afford a basis for an objective physical interpretation of the probabilities that figure in statistical descriptions of microscopic systems. They also imply that microscopic reversibility is not a property of finite 'closed' systems but only of the universe as a whole. But for this very reason they provide at best only a partial explanation of the thermodynamic arrow.

Borel's calculation shows that in a 'closed' gaseous system microinformation flows quickly into a very large number of external degrees of freedom. Such calculations can be used to justify assumptions about the initial absence of microinformation of the kind that figure in modern theories of irreversible processes. But the interaction between a system and its environment does not impose a particular direction on this flow of information, much less a common direction for all 'closed' systems. We are still faced with the paradox of a microscopically reversible* universe whose temporal structure, viewed macroscopically, is radically anisotropic.

\section{THE HISTORICAL ARROW}

So far I have discussed one aspect of this anisotropy, the thermodynamic arrow (defined by entropy-generating processes in 'closed' systems), and alluded to a second aspect, the cosmological arrow (defined by the cosmic expansion). But I have not mentioned what is perhaps the most conspicuous class of processes serving to define time's arrow: those that generate information in open systems. Such processes are central to all biological systems. They play an indispensable role in growth, in biological evolution, and in the phenomena of memory and consciousness. But they are not confined to living systems. A record of the Moon's past is written in its pitted surface; the internal structure of a star, like that of a tree, records the process of aging; and the complicated forms we observe in spiral galaxies reflect the evolutionary processes that shape them. The complex overlapping array of all these evolutionary records defines a third arrow of time, the historical arrow.

The thermodynamic arrow points in the direction of increasing entropy; the historical arrow points in the direction of increasing information. Equivalently, we may define the historical arrow through the statement that the present state of the universe (or of any sufficiently large subsystem of it) contains a partial record of the past but none of the future.

Because the thermodynamic arrow refers ideally to closed systems while the historical arrow manifests itself only in open systems, their coexistence presents no problem. Entropy-generating and information-generating processes normally proceed simultaneously and more or less independently in every complex system. In living systems, of course, harmful entropyproducing processes are usually offset by countervailing informationproducing processes.

\section{COSMOLOGY AND MACROSCOPIC PHYSICS}

I shall now sketch a theory that seeks to relate the three arrows of time--

* I am here neglecting the quantitatively small departures from microscopic time reversibility suggested by experiments on the decay of the $\mathrm{K}^{0}$ meson. 
the thermodynamic, the historical, and the cosmological - to one another, and to derive them all from a common postulate. This postulate-a slightly strengthened version of Einstein's cosmological principle--concerns the spatial structure of the universe. It states that no statistical property of the universe serves to define a preferred position or direction in space; the spatial structure of the universe is statistically homogeneous and isotropic*.

Before discussing the implications of this postulate, I should say a few words about the relationship between cosmology and macroscopic physics. From one point of view, cosmological theories are not essentially different from other physical theories. The cosmologist, like the astrophysicist, must make certain assumptions that cannot be verified directly; he must develop the consequences of these assumptions using relevant physical theories; and he must ultimately make predictions that are explicit enough so that they can be contradicted by appropriate observations or experiments. A good theory, whether in cosmology or any other branch of physics, enables one to draw more or less rigorous and quantitative inferences, in agreement with experience, from simple and natural assumptions - - which need not themselves be capable of direct verification. Thus the inaccessibility of stellar interiors to direct observation has not prevented the development of highly credible theories of stellar structure. Similarly, the impossibility of directly verifying postulates about the universe as a whole does not in itself doom cosmology to speculative status; the postulates themselves matter less than the quality and quantity of the inferences that can be drawn from them.

Yet there is a basic difference between cosmology and astrophysics. It stems from the fact that the universe is not one member of a class of similar objects characterized by a certain range of physical parameters, but is unique and all-embracing. The physical parameters that characterize a particular star, such as the Sun, have no special significance; other stars are more or less massive, have more or less angular momentum, are richer or poorer in metals. But while it is possible to construct mathematical models of the universe characterized by different sets of parameters, there is only one correct model. Hence its defining properties have special significance. In fact we may reasonably accord them the status of physical laws rather than auxiliary conditions.

If we adopt this point of view, we may reasonably employ the usual empirical criteria of simplicity and economy as guides in formulating appropriate cosmological postulates. The postulate of statistical homogeneity and isotropy seems to be the simplest assumption that does not conflict with any currently-accepted physical law.

\section{IMPLICATIONS OF THE COSMOLOGICAL PRINCIPLE}

Two well-known consequences of the cosmological principle are especially relevant to the present discussion.

First, the cosmological principle implies that the space-time continuum can be uniquely resolved into space plus time. For it is obvious that if statistical homogeneity and isotropy prevail in a given frame of reference,

\footnotetext{
* Einstein's cosmological principle, as it is normally employed, states that the universe can be
} represented, in a first approximation, by a uniform and isotropic distribution of matter. 
they cannot prevail in any frame of reference that is in motion--uniform or accelerated-with respect to this frame. The only symmetry-preserving transformations are spatial rotations and translations.

This conclusion may seem to have a paradoxical quality. Before Einstein, space and time were distinct. Special relativity fused them into a single four-dimensional continuum, replacing the concept of absolute space by that of the inertial frame of reference. Finally, through a further fusion of the concepts of gravitational force and inertial acceleration, general relativity succeeded in encompassing inertial and non-inertial frames of reference in a single mathematical formalism. The cosmological principle does not undo this work. Einstein's field equations still govern the local structure of a fourdimensional continuum in which space, time and gravitation are indissolubly blended; but at the cosmological level of description the old distinction between space and time re-emerges in new dress. And indeed it can be shown that the local inertial frame of reference- the frame in which Newton's theory is approximately valid-is one whose motion with respect to the preferred cosmological frame is unaccelerated. Thus the cosmological frame replaces absolute Newtonian space and time.

The second well-known consequence of the cosmological principle is the cosmic expansion. Einstein's equations (in the simplest and most widely accepted form of his theory) admit no static solutions satisfying the cosmological principle. The theory predicts that the universe expands (contracts) uniformly from (toward) a singular state of infinite density in the finite past (future). The rate of expansion depends on the equation of state and on the mean spatial curvature. For simplicity and definiteness, I shall assume in the following discussion that the spatial curvature is negative or zero. This implies that the universe is spatially infinite. A universe with positive spatial curvature, though unbounded has finite volume and is also, in a certain sense, finite in time. A discussion of this case would raise certain subtle and controversial points not essential to the main discussion.

\section{THE STRONG COSMOLOGICAL PRINCIPLE AND INDETERMINACY}

So far I have made use only of the weak form of the cosmological principle. The strong form, which postulates complete statistical homogeneity and isotropy, has a remarkable implication that does not seem to have been noted previously. Consider, by way of illustration, a statistically uniform (Poisson) distribution of points on a straight line; this is the simplest example of a statistically homogeneous (and isotropic) distribution. Let the line be divided into cells of equal length $h$, the analogues of quantum cells in sixdimensional phase space. The Poisson distribution is defined by a single parameter, $\bar{n}$, the mean occupation number of a cell. A particular realization of a given Poisson distribution is characterized by an infinite sequence of integer occupation numbers, e.g. ...00210111 ... Such realizations have a unique pair of properties, not shared by realizations of Poisson distributions on finite or semi-infinite segments. (a) From a single realization one can calculate the value of the statistical parameter $\bar{n}$ with arbitrary precision. This property, an immediate consequence of the law of large numbers, 
implies that a single realization contains all the statistical information needed to construct it. (b) Two realizations characterized by the same statistical parameter are operationally indistinguishable, since any finite sequence of occupation numbers must occur with precisely the same frequency in each sequence. Thus it is obviously impossible to devise an operational matching procedure that would distinguish between two realizations having the same statistical properties.

These properties of one-dimensional Poisson distributions obviously apply to statistically homogeneous distributions of points in six-dimensional phase space, provided that a suitably defined correlation distance is finite. (This property is needed to ensure the ergodicity of the distribution.) Let us agree to regard the values of quantities that figure in a complete statistical description of a statistically homogeneous and isotropic universe as constituting macroinformation, and all remaining information as microinformation. What has just been shown is that, under our assumptions, there is no microinformation; the uncertainties implicit in a statistical description of an infinite universe satisfying the strong cosmological principle are irreducible.

For example, if such a universe is in a state of thermodynamic equilibrium, it is completely characterized by its temperature and density; all other observable quantities can then be calculated. It is true that a hypothetical observer could measure the actual positions and velocities of molecules in a given region, but since all such regions are on exactly the same footing, and their statistical properties are calculable in advance, such measurements would convey no information in a technical sense.

The preceding argument depends essentially on the quantal character of the microscopic description. For in a classical (i.e. non-quantal) universe the distance between any two particles at a given instant serves to define a given statistical realization completely and to distinguish it from all other possible realizations of the same statistical description. Thus microinformation always exists in a classical universe.

The present conclusion goes beyond the implication of Borel's and similar calculations, that certain kinds of microinformation diffuse very rapidly from ostensibly closed systems. The diffusion process does not destroy information, it merely redistributes it. Thus the total quantity of microinformation in the universe remains constant. According to the present picture, however, microinformation is simply absent.

\section{GROWTH OF MACROINFORMATION IN COSMIC EVOLUTION}

Consider a universe filled uniformly with non-interacting particles. Let p denote the momentum of a particle as measured in a frame of reference that is locally at rest at the particle's instantaneous position. (The corresponding velocity $\mathbf{v}=c^{2} \mathbf{p} / E$ is thus the velocity relative to the uniformly expanding or contracting substratum.) The internal energy density $u$ and the temperature $T$ are defined by

$$
u=\frac{3}{2} n k T-n\langle E\rangle
$$

where $n$ denotes the mean particle density, and the particle energy $E=\sqrt{ }\left(m^{2} c^{4}+c^{2} p^{2}\right)$. An elementary kinematic calculation shows that the 


\section{DAVID LAYZER}

momentum of a free particle varies with time according to the simple law $\mathbf{p} \propto a^{-1}$, where $a$ is the cosmological scale factor defined by the relation $\rho a^{3}=$ constant. Thus the momentum of a free particle in an expanding universe continually decreases*. It follows that the temperature and the specific internal energy decrease with time in an expanding universe. In this important respect the universe does not behave like a closed system. We should therefore not be surprised to find that its thermodynamic behaviour differs from that of a closed system.

If the particles are ultra-relativistic (for example, if they are photons), $E \propto p$, so that $T \propto a^{-1}$. For non-relativistic particles on the other hand, $E \propto p^{2}$, so that $T \propto a^{-2}$. Thus, for a given rate of expansion, a relativistic gas cools less rapidly than a non-relativistic one.

Now consider a mixture of non-relativistic gas and radiation. Suppose that at some initial instant the mixture is in thermodynamic equilibrium at the temperature $T_{0}$. Suppose further that there is negligible interaction between the gas and the radiation. Then, as the universe expands (or contracts) away from the initial state, a temperature difference will develop between the two components $†$.

The cosmic expansion (or contraction) preserves the mean entropy per particle of each constituent, so that the specific entropy of the mixture does not change. But the maximum specific entropy increases monotonically in both directions of time. For if the thermalization rate were suddenly to become much greater than the expansion rate, the gas and the radiation would assume a common temperature, and in the process the specific entropy would increase. Hence at any given instant the actual specific entropy is less than its maximum possible value. In the general case, when the thermalization rate is neither vanishingly small nor infinitely large compared with the expansion rate, the specific entropy of the mixture will increase, but not as rapidly as maximum specific entropy. Since information is defined as the difference between the actual entropy and the maximum entropy (subject to given macroscopic constraints), this example shows that expansion or contraction from an initial state of thermodynamic equilibrium generates both specific entropy and specific information.

This conclusion obviously applies under much more general assumptions about the state and composition of the cosmic medium. The essential elements of the argument are (a) that the 'initial' state is one of maximum specific entropy (zero information), and (b) that the rate of cosmic expansion or contraction--which is of order $\sqrt{ }(6 \pi G \rho)$, where $\rho$ denotes the mean cosmic density - may be comparable to or greater than the rates of processes that tend to produce the state of local thermodynamic equilibrium. Because the cosmic expansion or contraction is not quasi-static, it generates departures from local thermodynamic equilibrium and hence generates information. At the same time, irreversible processes generate entropy.

* Since the frequency of a photon is proportional to its momentum, it diminishes with time in an expanding universe. This is the basis of the cosmological redshift-distance relation.

$\dagger$ It is easy to show that for extreme relativistic and extreme non-relativistic gases the thermal character of the momentum distribution is preserved under expansion or contraction. 
THE ARROW OF TIME

Suppose that at some epoch the universe was in a state of global thermodynamic equilibrium. Let us tentatively identify time's arrow with the direction in which cosmic entropy and information are generated, anticipating that this will turn out to coincide with the direction in which entropy and information are generated locally. Then the hypothesized state of thermodynamic equilibrium would indeed appear to be an initial state from which the universe must either expand or contract: the two possibilities are equally consistent with our considerations up to this point. Thus according to the present considerations, and in contrast with Dr Narlikar's conclusion, there is no direct link between the cosmic expansion and the thermodynamic arrow.

But we are not, of course, free to postulate that at some arbitrary epoch the universe was in a state of thermodynamic equilibrium. For such an assumption to be plausible, the cosmic expansion rate must be vanishingly small compared with local relaxation rates. A simple calculation shows that this condition is likely to be satisfied only near the singular state of infinite density. For it can be shown that the expansion rate $H \equiv \dot{a} / a \sim t^{-1}$ as $t \rightarrow 0(\rho \rightarrow \infty)$. On the other hand, two-body reaction rates vary as $n \mathrm{f}(T)$, where $n \propto a^{-3}$-at least for a certain range of values of $a$-and $\mathrm{f}(T)$ is a function of temperature. In a universe dominated by relativistic particles, $a \sim t^{\frac{1}{2}}$, while in one dominated by non-relativistic particles $a \sim t^{\frac{3}{3}}$. For interactions other than the Coulomb interaction, the two-body reaction rate is a non-decreasing - or at least not a rapidly increasing-function of temperature, which in turn is a decreasing function of time. It follows that in the limit $t \rightarrow 0$ the cosmic expansion rate does become infinitely slow compared with two-body reaction rates. It is therefore plausible to postulate that the cosmic expansion began from a state of near-thermodynamic equilibrium; the initial stages of the expansion are quasi-static, even though the expansion rate varies as $t^{-1}$.

To summarize the argument up to this point: From the assumption that no statistical property of the universe serves to define a preferred position or direction in space, we deduce that a complete description of the universe can be couched in statistical terms, and so contains no microinformation. On physical grounds, we postulate that thermodynamic equilibrium prevails in the limit $\rho \rightarrow \infty(t \rightarrow 0)$, and we define this as the initial state. Then macroscopic and microscopic information are both absent initially. The cosmic expansion generates entropy and information.

In a universe that expands from an initial singularity, every 'closed' system has a finite past and a more or less definite beginning in time. Given a sufficiently complete cosmogonic theory, one could in principle predict the statistical properties of all 'closed' systems and describe the processes by which they came or will come into being. In any case, a given 'closed' system will contain a certain quantity of macroinformation, but no microinformation (since none was present initially and the cosmic expansion generates only macroinformation, by definition). Thus the present theoretical scheme leads to precisely the sort of assumptions that have been introduced in modern theories of irreversible processes. And if this scheme could be developed into a quantitative theory, it would afford an explicit definition 
of macroinformation and macroscopic variables for every class of 'closed' systems.

In a general way, it is clear that the historical arrow must be related to the growth of information in the universe as a whole. In most open systems, however, the growth of information results directly from a redistribution of information within an effectively isolated parent system rather than from the cosmic expansion. But a detailed discussion of the historical arrow lies outside the scope of this article.

The scheme I have just outlined supports the intuitive ideas that the world is unfolding in time and that the future is never wholly predictable. For, as we have seen, the specific information content of the universe increases steadily in the direction away from the initial state of maximum cosmic density. This implies that the present state of the universe cannot contain enough information to define any future state. The future grows from the past, as a plant grows from a seed, yet it contains more than the past.

\section{COSMIC EVOLUTION}

Can the kind of cosmological assumptions I have discussed support a theory of cosmic evolution that accounts for the observed properties of the astronomical universe?

Having postulated an initial state of thermodynamic equilibrium, we are left with just one free parameter at our disposal: the temperature at a given epoch or density. Fixing this parameter involves a choice between two main possibilities, usually referred to as the hot universe and the cold universe.

In the hot universe, energy resides chiefly in electromagnetic radiation during the early stages of the expansion. The cosmic microwave background (which is thought to result from a thermal radiation field with $T=2.7^{\circ} \mathrm{K}$ ) is interpreted as a remnant of the primordial radiation field; this allows the initial temperature to be calculated. One can then go on to calculate the relative abundances of elements heavier than hydrogen that would be formed during the early stages of the cosmic expansion. The most crucial prediction is that of the helium abundance, which turns out to be about 28 per cent ${ }^{5}$. Observational tests of this prediction are extremely difficult. There is some evidence for the existence of stars whose atmospheres contain substantially less than the predicted primeval abundance, but astronomers do not yet accept it as conclusive ${ }^{6}$.

The stability of the hot universe against local density fluctuations has been carefully studied by a number of authors, with consistently negative results. If only thermal fluctuations were present when the cosmic medium had the density of a nuclear fluid, significant local inhomogeneities could never have developed. This conclusion has forced proponents of the hot universe to postulate that substantial density fluctuations either were present initially or were created at supernuclear densities by physical processes whose experimental verification lies outside the scope of current experimental techniques.

The alternative approach is to postulate an initial state cold enough to allow the formation of substantial density fluctuations. One must then find an alternative explanation for the cosmic microwave background. The 
requirements for such an explanation imposed by the observed quantity and quality of the radiation field are rather stringent, but can perhaps be satisfied by a hypothesis that links the background radiation field to large-scale explosive events occurring within galaxies during their formative stages ${ }^{7}$.

If the initial temperature is sufficiently low, the universe may freeze into solid molecular hydrogen--a possibility first suggested by Zeldovich ${ }^{8}$. Continued expansion would cause the solid cosmic medium to shatter into fragments, whose masses, as a simple calculation shows, would be comparable to those of planetary satellites.

I have developed an approximate and somewhat speculative theory of the ensuing evolutionary stages ${ }^{9}$. The 'gas' composed of solid-hydrogen fragments is unstable against a form of turbulence driven by local gravitational forces. The turbulence creates a wide spectrum of density fluctuations. Ultimately self-gravitating systems begin to separate out. The least massive systems separate out first, then clusters of these systems, then clusters of these clusters, and so on. In this way a hierarchy of self-gravitating systems comes into being, and is still in process of formation at the present time.

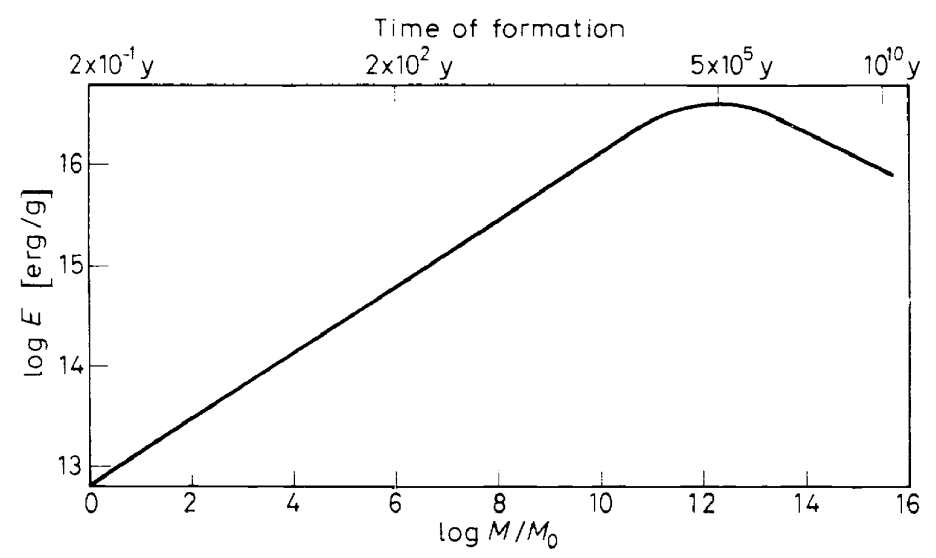

Figure 1. Specific binding energy versus mass for newly formed self-gravitating systems, according to an approximate cosmogonic theory (ref. 9).

Although the theory is speculative and approximate, it predicts a definite relation between the average binding energy per unit mass and the mass of newly formed self-gravitating systems, shown in Figure 1. The most tightly bound systems correspond to galaxies and compact galaxy clusters, which accordingly are predicted to have masses around $10^{12}$ solar masses and binding energies around $10^{16} \mathrm{erg} / \mathrm{g}$. These and other semi-quantitative predictions agree surprisingly well with observational estimates over a mass range of more than fifteen decades. These tentative and approximate results encourage one to believe that further theoretical studies along the lines I have sketched may ultimately lead to a quantitative understanding of cosmic evolution as well as a qualitative understanding of time's arrow. 


\section{DAVID LAYZER}

\section{REFERENCES}

1 A. Einstein, Phys. Z. 10, 185 (1909).

${ }^{2}$ L. van Hove, Physica, 21, 512 (1955).

${ }^{3}$ For example, I. Prigogine, Non-equilibrium Statistical Mechanics, Interscience: New York (1962).

W. Kohn and M. Luttinger, Phys. Rev. 108, 590 (1957); 109, 1892 (1958).

M. Kac, Proceedings of the Third Berkeley Symposium, (J. Neyman, ed.), Vol. III, p 171. University of California Press : Berkeley (1956).

4 E. Borel, Introduction Géométrique à la Physique, Gauthier-Villars : Paris (1912).

E. Borel, Introduction Géométrique à Quelques Théories Physiques, Gauthier-Villars: Paris (1914).

See also J. M. Blatt, Prog. Theor. Phys. 22, 745 (1959).

P. Morrison, Preludes in Theoretical Physics, (edited by A. de-Shalit, H. Feshbach and L. van Hove), p 347. North-Holland: Amsterdam (1966).

P. G. Bergmann and J. L. Lebowitz, Phys. Rev. 99, 578 (1955).

5 R. V. Wagoner, W. A. Fowler and F. Hoyle, Astrophys. J. 148, 3 (1967). This paper contains references to earlier and less complete calculations.

6 A. comprehensive review of this question is given by I. J. Danziger, Annu. Rev. Astron. Astrophys. 8 (1970).

7 D. Layzer, Astrophys. Letters, 1,99 (1968).

8 Y'a. B. Zel'dovich, J. Exp. Theor. Phys. 16, 1102 and 1395 (1963).

9 D. Layzer, 'Cosmogonic Processes,' Proceedings of the Brandeis Summer Institute in Theoretical Physics-1968 (to be published). 